\title{
Uncertainty-dependent activity within the ventral striatum predicts task-related changes in response strategy
}

\author{
George A. Buzzell ${ }^{1,2}$ (D) Daniel M. Roberts ${ }^{1,2}$ - John R. Fedota ${ }^{1,2}$. \\ James C. Thompson ${ }^{1,2} \cdot$ Raja Parasuraman ${ }^{1,2}$ - Craig G. McDonald ${ }^{1,2}$
}

Published online: 9 October 2015

(C) Psychonomic Society, Inc. 2015

\begin{abstract}
Recent neuroimaging work has demonstrated that the ventral striatum (VS) encodes confidence in perceptual decisions. However, it remains unclear whether perceptual uncertainty can signal the need to adapt behavior (such as by responding more cautiously) and whether such behavioral changes are related to uncertainty-dependent activity within the VS. Changes in response strategy have previously been observed following errors and are associated with both medial frontal cortex (MFC) and VS, two components of the performance-monitoring network. If uncertainty can elicit changes in response strategy (slowing), then one might hypothesize that these changes rely on the performancemonitoring network. In the present study, we investigated the link between perceptual uncertainty and task-related behavioral adaptations (response slowing and accuracy increases), as well as how such behavioral changes relate to uncertainty-dependent activity within MFC and VS. Our participants performed a two-choice perceptual decision-making task in which perceptual uncertainty was reported on each trial while behavioral and event-related functional magnetic resonance imaging data were collected. Analysis of the behavioral data revealed that uncertain (but correct) responses led to slowing on subsequent trials, a phenomenon that was positively correlated with increased accuracy. Critically, postuncertainty slowing was negatively correlated with the VS activity elicited by uncertain responses. In agreement with
\end{abstract}

George A. Buzzell

gbuzzell@gmu.edu

1 Department of Psychology, George Mason University, 4400 University Drive MS 3F5, Fairfax, VA 22030, USA

2 Center of Excellence in Neuroergonomics, Technology, and Cognition (CENTEC), Fairfax, VA, USA previous reports, increases in MFC activation were observed for uncertain responses, although MFC activity was not correlated with post-uncertainty slowing. These results suggest that perceptual uncertainty can serve as a signal to adapt one's response strategy and that such behavioral changes are closely tied to the VS, a key node in the performance-monitoring network.

Keywords Uncertainty · Ventral striatum · Cognitive control . Post-trial slowing $\cdot$ Post-error slowing $\cdot$ Perceptual decision-making

Recent research has begun to identify the neural underpinnings of perceptual uncertainty (Fleck, Daselaar, Dobbins, \& Cabeza, 2006; Fleming, Huijgen, \& Dolan, 2012), with the ventral striatum (VS) encoding a choice-independent confidence signal (Hebart, Schriever, Donner, \& Haynes, 2014). However, the relationship between the neural basis of perceptual uncertainty and the ongoing regulation of task performance remains unclear. For example, when performing a difficult task, do participants not only slow down on uncertain (but correct) responses, but also slow down and become more cautious in their response strategy on subsequent trials? If so, do these adaptive behavioral changes relate to a confidence signal encoded within the VS? Investigations of how individuals change their behavior following error commission have been well studied within the performance-monitoring literature; participants typically slow their responses following errors (Rabbitt \& Phillips, 1967), a phenomenon that is believed to reflect increased cautiousness (Botvinick, Braver, Barch, Carter, \& Cohen, 2001; Dutilh et al., 2011). However, whether perceptual uncertainty on correct trials can also signal the need to adapt ongoing behavior remains unclear. 
Previous work investigating perceptual uncertainty has shown that increasing uncertainty is associated with increased activation within the medial frontal cortex (MFC; Fleck et al., 2006; Fleming et al., 2012; Hebart et al., 2014) and reduced activity within the VS (Hebart et al., 2014). That is, perceptual uncertainty appears to modulate activity within key nodes of the performance-monitoring network. Additionally, recent work has demonstrated that uncertain responses are rated as "less pleasant," or rather, aversive in nature (Clos, Schwarze, Gluth, Bunzeck, \& Sommer, 2015; Schwarze, Bingel, Badre, $\&$ Sommer, 2013), and there is evidence that events (such as errors) that trigger behavioral adaptations due so, at least in part, as a result of their aversive nature (Botvinick, 2007; Dreisbach \& Fischer, 2012; Holroyd \& Coles, 2002; Shenhav, Botvinick, \& Cohen, 2013). Taken together with similar activations within the performance-monitoring network, the aversive quality of uncertainty may cause behavioral adaptations similar to those seen following errors.

A recent report by Hebart et al. (2014) demonstrated that the VS encodes the choice-independent confidence of perceptual decisions, and the authors speculated that this confidence signal may be used to regulate ongoing behavior. Similarly, previous work using episodic memory tasks has shown that confidence is associated with elevated VS activity (Clos et al., 2015; Schwarze et al., 2013) and that the amount of activation within the VS on correct trials predicts task performance across individuals in a working memory task (Satterthwaite et al., 2012). The notion that the VS may play a direct role in task performance or behavioral adaptations is in line with previous work demonstrating that VS activity predicts intrinsic motivation (Murayama, Matsumoto, Izuma, \& Matsumoto, 2010) or the amount of effort invested in obtaining a reward (Schmidt, Lebreton, Cléry-Melin, Daunizeau, \& Pessiglione, 2012). Although the performance-monitoring literature typically interprets VS activity in terms of an input to the MFC (Holroyd \& Coles, 2002; Shenhav et al., 2013), with the MFC directly predicting behavioral adaptations, it is important to note that the VS also receives extensive projections from the MFC (Haber \& Knutson, 2010). Additionally, the VS forms reciprocal connections with other midbrain structures and, through the nucleus basalis and globus pallidus, it has access to cortical and motor regions (Groenewegen, Wright, \& Beijer, 1996; Haber \& Knutson, 2010). Thus, it is conceivable that confidence (or uncertainty) encoded within the VS may contribute to behavioral adaptations, either through the MFC (similar to the changes seen following errors) or through other neural pathways.

Post-error slowing (PES) and post-error increases in accuracy (PEIA) are two of the most well-studied forms of behavioral adaptations following errors. Traditionally, PES has been interpreted as a form of top-down control, reflecting increased cautiousness (Botvinick et al., 2001) and an elevated decision threshold (Ratcliff \& McKoon, 2008) adopted to prevent further errors. It has been shown that PES correlates with MFC activity, suggesting that the MFC is related to the instantiation of control following errors (Chevrier \& Schachar, 2010; Garavan, Ross, Murphy, Roche, \& Stein, 2002; Kerns et al., 2004; Klein et al., 2007), although a direct relationship between MFC and PES is not always observed (Danielmeier, Eichele, Forstmann, Tittgemeyer, \& Ullsperger, 2011; King, Korb, von Cramon, \& Ullsperger, 2010). As has been noted previously, the finding that uncertainty elicits MFC activity and, like errors, is associated with negative valence, suggests that uncertain responses may also lead to post-trial slowing. If this is the case, it seems reasonable to suggest that, similar to the PES-MFC relationship previously observed, postuncertainty slowing might also correlate with MFC activity. However, a growing literature also suggests a direct link between the VS and confidence, and it has been suggested that the VS may play a role in influencing task performance (Hebart et al., 2014; Satterthwaite et al., 2012). Therefore, if uncertainty elicits behavioral adaptations, such as slowing responses on subsequent trials, it remains unclear whether such adaptations would be directly related to MFC activation or more closely associated with VS activity.

Taken together, both the performance-monitoring literature and complementary research illustrating the relationship between the VS and motivation or confidence suggest a link between uncertainty and behavioral adaptations. The present study was designed to explicitly test whether uncertainty engages behavioral adaptations and, if so, through which neural substrate(s) these adaptations are instantiated. Participants performed a difficult perceptual discrimination task while certainty was assessed on each trial. On the basis of previous work (Fleck et al., 2006; Fleming et al., 2012; Hebart et al., 2014), we expected uncertainty to be associated with increased MFC and reduced VS activity. Additionally, we assessed whether uncertainty leads to slowing and/or increased accuracy on subsequent trials and how these behavioral adjustments relate to activity within the VS and MFC.

\section{Method}

\section{Participants}

Twenty-six participants were recruited from the George Mason University student population and paid $\$ 15 / \mathrm{h}$ for their participation. Due to low behavioral accuracy or excessive motion during functional magnetic resonance imaging (fMRI) acquisition, two participants were removed from further analysis, leaving 24 participants in the final analysis (13 female, 11 male; mean age $=22.96, S D=2.74)$. All of the participants were right-handed, had normal (or corrected-tonormal) vision, had no known neurological deficits, and were 
not currently taking any medications known to affect the central nervous system. All participants provided written informed consent, and all procedures were approved by the George Mason University Office of Research Integrity and Assurance.

\section{Experimental task}

Participants completed a two-handed, forced choice stimulus discrimination task in which vertical Gabor patches had to be distinguished from nonvertical Gabor patches (see Fig. 1). On each trial, participants provided both their perceptual choice and their confidence in their response (choice certainty). The task difficulty was individually calibrated to $\sim 70 \%$ accuracy through a psychophysical staircase procedure completed prior to the experimental task. On each trial, stimuli were presented for $200 \mathrm{~ms}$, and participants responded with one hand for vertical stimuli and with the other hand for nonvertical stimuli (counterbalanced across participants). Additionally, participants were instructed to use their index and middle fingers to indicate either a "sure" or an "unsure" response (counterbalanced across participants) using a pair of MRIsafe button boxes (see Fig. 1). In this way, both accuracy (which hand was used) and certainty (which finger was used) were obtained on every trial with a single response. In order to place an emphasis on the certainty of stimulus identity, and not aspects of error monitoring, participants were instructed to focus on "how sure you were of the stimulus identity, and not whether you thought you were correct or not." Additionally, to remove the confound of unintentional-

A
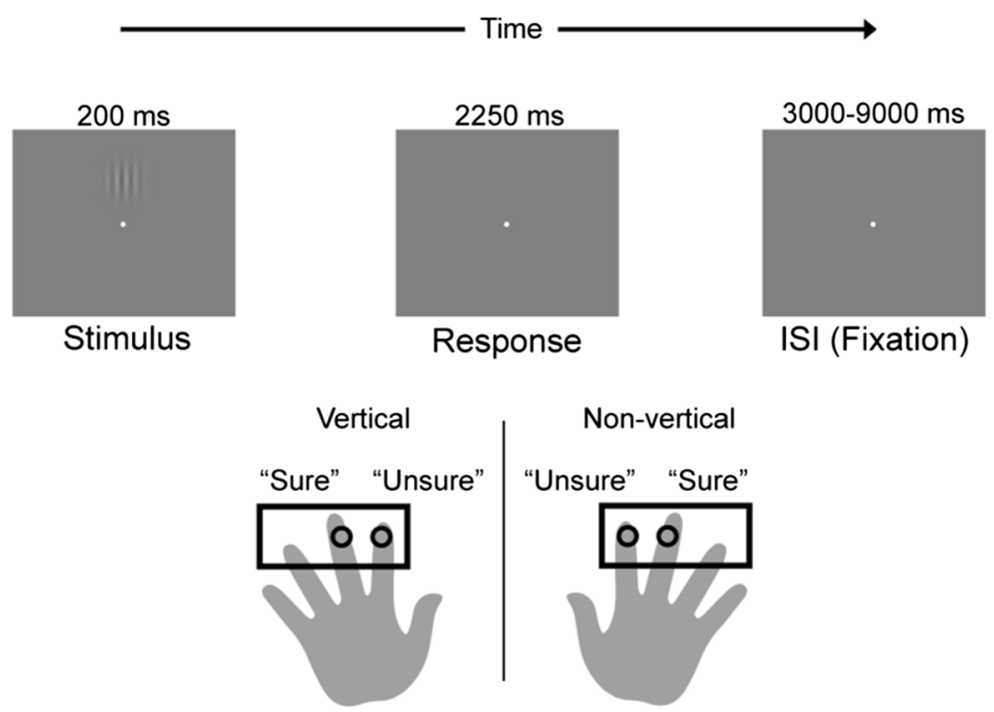

B

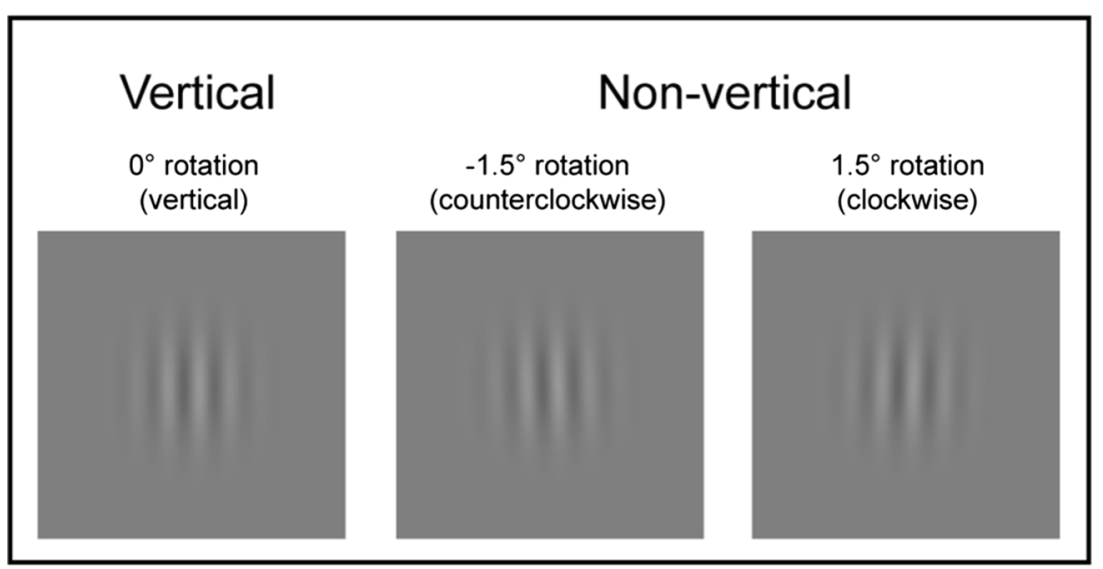

Fig. 1 Experimental task and stimuli. a Depiction of the experimental task. Participants were required to discriminate between vertical and nonvertical Gabor patches and to indicate their perceptual confidence. The perceptual choice was indicated with a hand response (e.g., left hand for vertical stimuli), and certainty was indicated with a finger response (e.g., index finger for "unsure" responses). Both the choice- hand and certainty-finger response mappings were counterbalanced across participants. b Average rotation values for vertical and nonvertical Gabor stimuli. The actual stimuli were created on an individual basis, in order to achieve $\sim 70 \%$ accuracy during the experiment 
response errors, participants were allowed to correct their responses if they had pressed a response key "by accident," but not if they "changed their mind." Any trials in which participants corrected their responses were removed from further analysis. Participants were instructed to respond as quickly and accurately as possible and were provided with a 2,250$\mathrm{ms}$ response window in which to make their response. If participants responded after the 2,250-ms response deadline, a "too slow" message appeared on the screen and this trial was removed from further analyses. No feedback regarding choice accuracy was provided during the task. Following each trial, a mean interstimulus interval (ISI) of $4.8 \mathrm{~s}$ (drawn from a gamma distribution, ranging from 3 to $9 \mathrm{~s}$ ) preceded the presentation of the following trial. Participants completed nine blocks (48 trials each, 432 trials total) of the task while fMRI data were acquired. The total time for the experimental task was $\sim 50 \mathrm{~min}$.

\section{Stimuli}

The stimuli used for the experimental task were vertical and nonvertical (rotated approximately $1.5 \mathrm{deg}$ from center), lowcontrast Gabor stimuli (see Fig. 1). The exact rotation for the nonvertical stimuli was calibrated on an individual basis, using a psychophysical staircase procedure. Gabor stimuli were generated using the MATLAB programming environment (MathWorks, Natick, MA), the Psychophysics Toolbox functions (Brainard, 1997; Kleiner, Brainard, \& Pelli, 2007; Pelli, 1997), and custom MATLAB scripts. The stimuli consisted of a sinusoidal grating measuring three cycles per degree, a Gaussian mask spanning 1.6 cycles per standard deviation, and a peak contrast level of $20 \%$. The Gabor stimuli subtended approximately $2 \times 2$ deg and were presented on a gray background, 1.5 deg above a white fixation point subtending $0.3 \mathrm{deg}$ in diameter. Both vertical and nonvertical Gabor stimuli appeared with $50 \%$ probability, with nonvertical stimuli additionally being divided into an equiprobable number of stimuli rotated clockwise or counterclockwise.

\section{Psychophysical staircase procedure}

A psychophysical staircase procedure was completed prior to the experimental task (on a separate day) in order to identify the stimulus rotation that would achieve approximately $70 \%$ accuracy for each participant. The task parameters for the staircase procedure were identical to those for the experimental task, with the exception of confidence not being reported on each trial. During the staircase procedure, if participants responded correctly on two consecutive trials, the rotation value of the nonvertical Gabor stimuli was decreased (made more difficult) by $0.25 \mathrm{deg}$. If participants responded incorrectly on any trials, the rotation of the nonvertical Gabor stimuli was increased (made easier) by $0.25 \mathrm{deg}$. In this way, the staircase procedure settled on the exact rotation value that would elicit approximately $70 \%$ accuracy for each participant $(M=1.50 \mathrm{deg}, S D=0.44 \mathrm{deg})$. The staircase procedure took approximately $5 \mathrm{~min}$ to complete and was run twice to ensure proper calibration.

\section{Task practice prior to the scanning session}

Approximately one week prior to the scanning session, participants completed the psychophysical staircase procedure and received training on the experimental task. Following the task calibration, participants practiced the experimental task (48 trials, with feedback), followed by three blocks (48 trials each) of the experimental task (without feedback). Given concerns over an adequate signal-to-noise ratio for the BOLD response in all conditions of interest (correct-sure, correct-unsure, errors), participants were only asked to participate in the MRI task if their behavioral results suggested adequate representation of all experimental conditions of interest. That is, each condition of interest needed to account for at least $16 \%$ of the responses. When participants arrived at the MRI scanning suite approximately one week later, they practiced one block of the experimental task (48 trials, with feedback) prior to beginning the scanning session.

\section{Behavioral analysis}

For all analyses, trials on which participants did not respond or responded before $150 \mathrm{~ms}$ or after the 2,250-ms response deadline were removed from further analysis. In addition, trials on which participants accidentally pressed the wrong button and subsequently corrected their response were removed from the analysis. The removed trials made up less than $6 \%$ of the data.

The effect of certainty on the accuracy and response time (RT) data was analyzed both as a function of the current trial $(t)$ and as a function of the previous trial $(t-1)$, allowing for investigations of both the overall effect of certainty and the contextual effects of certainty. Analysis of the relationship between certainty and RT was investigated using a two-way, previous trial (correct-sure, correct-unsure) by current trial (correct-sure, correct-unsure) repeated measures analysis of variance (ANOVA). In order to compare this analysis with our primary analysis of the imaging data, the analysis of certainty data was restricted to correct trials only. Additionally, in order to investigate the relationship between certainty and accuracy (as an outcome measure), we employed a similar two-way, repeated measures ANOVA, with previous trial (correct-sure, correct-unsure) and current trial (sure, unsure) as factors. We additionally tested whether post-uncertainty slowing (PUS) was correlated with post-uncertainty increases in accuracy (PUIA) by computing a Pearson product-moment correlation, based on the assumption that a positive correlation would suggest that PUS is adaptive, in that it leads to increases 
in task performance. For the correlation between PUS and PUIA, PUS was defined as the percentage change in the RT for correct-sure trials following correct-unsure trials, relative to each individual's baseline RT for correct-sure trials following correct-sure trials. Similarly, PUIA was defined as the percentage change in accuracy for sure trials following correct-unsure trials, relative to each individual's baseline accuracy for sure trials following correct-sure trials.

In addition to analyzing the contextual effects of certainty, we also analyzed the contextual effects of accuracy (collapsed across certainty) as a more traditional analysis of PES and PEIA. The effects of the previous- and current-trial accuracy on RT were assessed with a two-way, previous trial (correct, error) by current trial (correct, error) repeated measures ANOVA. Accuracy changes (PEIA) were analyzed using a paired-samples $t$ test comparing the mean accuracy for postcorrect versus post-error trials.

The present study was designed to ensure adequate representation of the three primary conditions of interest (correctsure, correct-unsure, errors), and the primary analysis of the behavioral data focused on these conditions (described above). However, in order to more fully explore the behavioral data, we also conducted an exploratory analysis that not only incorporated the effects of current- and previous-trial certainty, but also current- and previous-trial accuracy in one model. Although this exploratory analysis could only be performed for a subset of the participants $(n=18)$ who had trials in all cells, it allowed for testing whether the contextual effects of certainty interacted with the contextual effects of errors.

The effects of both certainty and accuracy on RT were investigated using a four-way, previous-trial certainty (sure, unsure) by previous-trial accuracy (correct, error) by currenttrial certainty (sure, unsure) by current-trial accuracy (correct, error) repeated measures ANOVA. Additionally, in order to investigate the effects of certainty and accuracy on the currenttrial accuracy (as an outcome measure), we employed a threeway, previous-trial certainty (sure, unsure) by previous-trial accuracy (correct, error) by current-trial certainty (sure, unsure) repeated measures ANOVA. No correlations were tested in association with these exploratory analyses, since the number of participants $(n=18)$ would be too low to detect any reliable correlations.

\section{Imaging acquisition and preprocessing}

The fMRI data were acquired using a Siemens Allegra 3-T scanner, equipped with a one-channel birdcage head coil. During each run, $\mathrm{T} 2 *$ gradient-echo, echo-planar imaging was acquired, with a repetition time/echo time (TR/TE) of 2 , $300 / 30 \mathrm{~ms}$, flip angle $=90 \mathrm{deg}, 40$ interleaved axial slices $3 \mathrm{~mm}$ thick/.3-mm gap, field of view $=192 \mathrm{~mm}$, and matrix size $=64 \times 64$ (in-plane resolution of $\left.3 \mathrm{~mm}^{2}\right)$. An average of 121 time points (TRs) were acquired per run, and nine runs were acquired for each participant. Following fMRI acquisition, two T1, whole-head, structural scans were acquired using a three-dimensional, magnetization-prepared, rapidacquisition gradient echo (MPRAGE) pulse sequence. Two $\mathrm{T} 1$ scans were collected to ensure that at least one artifactfree T1 was obtained for each participant; during the analysis, only one $\mathrm{T} 1$ scan was used for the registration of each participant's data. During the MPRAGE sequence, 160 1-mm-thick slices $(256 \times 256$ matrix, field of view $=260,0.94$ $\mathrm{mm}$ voxels) were acquired with a TR/TE of $2,300 / 3 \mathrm{~ms}$.

All analyses of the fMRI data were performed using FSL (www.fmrib.ox.ac.uk/fsl). In order to allow the scanner to reach its equilibrium magnetization, the first five volumes were removed prior to analysis. The fMRI data were highpass filtered (128-s cutoff), slice-timing corrected (Hanningwindowed sync interpolation to shift each time series relative to the middle of the TR period), and motion corrected using FMRIB's Linear Registration Tool (FLIRT). For two of the participants, a single run had to be removed due to excessive motion above $1 \mathrm{~mm}$. Prewhitening using FMRIB's improved linear model was performed to remove temporal autocorrelation in the fMRI time series data. The data were smoothed using a 6-mm full-width at half-maximum Gaussian kernel. Coregistration was completed in a two-step process: The functional data were first registered to a high-resolution structural image (MPRAGE) using FLIRT following brain extraction using the Brain Extraction Tool (BET) with the fractional intensity threshold set to .35 . Registration to the standard space (T1 2-mm MNI template) was then performed using FMRIB's Nonlinear Registration Tool and a warp resolution of $10 \mathrm{~mm}$.

\section{fMRI analysis}

Primary analysis of imaging data The present study was designed to ensure adequate trial counts for three trial types of interest (correct-sure, correct-unsure, and errors), allowing for investigation of the effects of certainty when no explicit errors had been made (correct-sure vs. correct-unsure), as well as for a traditional comparison of error and correct responses (collapsed across certainty). For this reason, the primary analysis of the imaging data utilized a general linear model (GLM), with three regressors of interest to separately model the onsets of correct-sure, correct-unsure, and error trials. For this primary analysis, errors were not split into error-sure and error-unsure responses, given the low trial counts for the errorsure condition in a majority of the participants. Planned contrasts were used to investigate the effect of certainty (correctsure vs. correct-unsure) and the effect of accuracy (correct vs. error).

For each of the nine runs, three task-related regressors were convolved with a canonical double-gamma hemodynamic response function (HRF) with no phase delay. As in the 
behavioral analysis, trials with no response were excluded, as well as those on which participants responded before 150 or after 2,250 ms, or on which responses were corrected; the excluded trials made up less than $6 \%$ of the data. Six motion parameters (three translation, three rotation) were also added to the GLM as confound regressors in order to account for residual motion effects after correction by FLIRT (nine regressors total). For three participants who had between one and two runs with relatively few movement artifacts above $1 \mathrm{~mm}$, FMRIB's fsl_motion_outlier tool was used to ignore these time points for the GLM. A second-level analysis was used to average across the nine runs for each participant using a fixed-effects model. The data were than averaged across participants in a third-level analysis, using FMRIB's Local Analysis of Mixed Effects (FLAME1). For the third-level analysis, the within-subjects variance for the three regressors of interest (correct-sure, correct-unsure, and errors) was modeled using fixed effects, and participant (betweensubjects variance) was modeled as a random effect. Separate contrasts comparing the effects of certainty (for correct trials only) and accuracy (collapsed across certainty) were performed. For all contrasts of the primary analysis, the familywise error rate (FWER) was controlled using a cluster-based correction based on Gaussian random field (GRF) theory $(Z=$ $2.3, p=.05)$.

Correlations between behavioral and imaging data As we describe in the Results section, the group-level contrast comparing sure and unsure responses (for correct trials only) revealed uncertainty-dependent activations within the MFC and VS. Additionally, analysis of the behavioral data revealed a significant PUS effect (also described in the Results section). On the basis of these findings, we then sought to identify whether either of the uncertainty-dependent brain regions (MFC, VS) correlated with behavioral adaptations (PUS). In order to identify whether the MFC or VS activations were correlated with PUS, we used the MFC and VS clusters identified in the group-level fMRI analysis as regions of interest (ROIs) to test for correlations between individuals' brain activity and PUS. For the MFC ROI, the mean percent signal change from the correct-unsure $>$ correct-sure contrast was extracted for each participant (i.e., MFC increases) using the FSL featquery tool. Similarly, for the VS ROI the mean percent signal change from the correct-unsure $>$ correct-sure contrast was extracted for each participant (i.e., VS decreases). These values were then separately tested for Pearson product-moment correlations with the PUS effect, defined as the percentage change in RT for correct-sure trials following correct-unsure trials, relative to each individual's baseline RT for correct-sure trials following correct-sure trials. In this way, we tested whether increases in the MFC or decreases in the VS for uncertain responses were correlated with the degree to which participants slowed down following perceptually unsure responses. We chose to employ an ROI-based approach given that we were explicitly interested in whether the regions that were modulated by uncertainty also correlated with PUS.

Within-subjects analysis of the slowing effect Following investigation of a link between uncertainty-dependent brain activity and post-uncertainty slowing at the group level (between subjects), we then sought to investigate whether similar brain regions were also predictive of slowing at the withinsubjects level. For this within-subjects analysis, an approach similar to that of Kerns et al. (2004) was used, in which events of interest were modeled separately at the subject level, on the basis of whether the following trial's RT value was above or below the median RT for the condition of interest. This second GLM was largely similar to the primary analysis of the imaging data, with three important differences. (1) In the first-level analysis, correct-unsure responses were separately modeled on the basis of whether they were followed by above- or below-median RT values on the following trial; three additional regressors were included to account for above- and belowmedian correct-sure responses, as well as error trials (five regressors total). (2) Not only were bad trials (those in which participants responded before $150 \mathrm{~ms}$ or after $2,250 \mathrm{~ms}$, or did not respond at all) excluded, but the trials immediately following bad trials were also excluded. (3) Separate contrasts comparing the effects of slowing (for either unsure or sure trials) were performed. Given that the primary analysis had identified the VS and MFC as brain regions that exhibited uncertainty-dependent activations, all contrasts for this secondary analysis were performed within $20-\mathrm{mm}$ spherical ROIs centered on the left and right nucleus accumbens (NAcc), as well as the MFC (centered on the region of MFC sensitive to performance monitoring, as defined by the metaanalysis of Klein et al., 2007). Within each ROI, the FWER was controlled using a cluster-based correction based on GRF theory $(Z=2.3, p=.05)$.

Exploratory analysis of the certainty by accuracy interaction The primary analysis of the imaging data investigated the effect of certainty within correct trials only, as well as the comparison of correct and incorrect responses. However, the primary analysis did not investigate any potential interactions between certainty and accuracy. For example, it is possible that the effect of certainty might only be present within correct trials and would be absent within error trials. Testing whether certainty and accuracy interact requires parsing error trials into error-sure and error-unsure responses, and given the low trial counts for the error-sure condition in a majority of participants, this analysis was not run in the primary analysis. However, in order to explore the potential interaction between certainty and accuracy within the imaging data, an exploratory analysis using a novel GLM was also conducted. 
This third GLM was largely similar to the primary analysis of the imaging data, with four important differences. (1) In the first-level analysis, four separate regressors were used to model correct-sure, correct-unsure, error-sure, and error-unsure responses; for runs in which an error-sure response did not occur, this fourth regressor was left out of the model. (2) At the second level, a fixed-effects model was used to average across all runs in which each of the four regressors of interest were modeled (correct-sure, correct-unsure, and error-unsure trials were modeled in all runs for all participants, whereas error-sure trials were not modeled in some runs because this event did not occur). (3) In the third (group-level) analysis, the main effect of certainty, the main effect of accuracy, as well as the interaction between accuracy and certainty were all modeled as fixed effects, while participant (between-subjects variance) was modeled as a random effect. (4)Separate contrasts comparing the main effect of certainty, the main effect of accuracy, and the certainty by accuracy interaction were performed. Given that the primary analysis had identified the VS and MFC as uncertainty-dependent brain regions, all contrasts for this third analysis were performed within 20-mm spherical ROIs centered on the left and right NAcc, as well as the MFC (centered on the region of MFC sensitive to performance monitoring, as defined by the meta-analysis of Klein et al., 2007). Within each ROI, the FWER was controlled using a cluster-based correction based on GRF theory $(Z=2.3, p=.05)$. In order to better illustrate the effects present (or absent) in this third analysis, plots of the percent signal change within the right VS (6-mm sphere centered on the right NAcc), left VS (6-mm sphere centered on the left NAcc), and MFC (6-mm sphere centered on the region of MFC sensitive to performance monitoring, as defined by the meta-analysis of Klein et al., 2007) for correctsure, correct-unsure, error-sure, and error-unsure trials, were extracted using the FSL featquery tool and then plotted.

\section{Results}

\section{Behavioral results}

In order to study the effects of perceptual uncertainty, the experimental task was designed to be highly difficult and to elicit a large number of uncertain responses. Accordingly, the mean accuracy was $74.36 \%(S E=1.84 \%)$, with correct responses being approximately equally split between reports of being sure and unsure (unsure: $M=51.41 \%, S E=2.68 \%$ ) and errors being dominated by unsure responses $(M=$ $65.29 \%, S E=3.56 \%$ ).

Analysis of the RT data as a function of certainty (for correct trials only) revealed a main effect of certainty $[F(1,23)=$ $52.71, p<.001]$, with participants responding more slowly when they reported being unsure rather than sure (see Table 1). Additionally, a significant current- by previous-trial
Table 1 Mean response time (RT) and accuracy as a function of previous trial type

\begin{tabular}{lll}
\hline Current Trial & \multicolumn{2}{l}{ Previous Trial } \\
\cline { 2 - 3 } & Correct-Sure & Correct-Unsure \\
\hline Correct-sure RT (ms) & $880.26(29.72)$ & $925.15(34.11)$ \\
Correct-unsure RT (ms) & $1,034.02(37.30)$ & $1,003.14(36.63)$ \\
Sure accuracy & $79.69 \%(2.78 \%)$ & $80.87 \%(2.49 \%)$ \\
Unsure accuracy & $70.73 \%(1.66 \%)$ & $70.28 \%(22.16 \%)$ \\
\hline
\end{tabular}

Standard errors of the means are shown in parentheses

interaction was identified $[F(1,23)=18.67, p<.001]$. Follow-up comparisons revealed a significant PUS effect, with participants responding more slowly on correct-sure trials that followed correct-unsure responses than on correct-sure trials that followed correct-sure responses $[t(1,23)=-3.40, p$ $=.002]$. Additionally, participants responded more quickly on correct-unsure trials when they followed correct-unsure responses than when they followed correct-sure responses $[t(1$, $23)=2.70, p<.013]$. See Table 1 and Fig. 2 a for depictions of the RT data as a function of current- and previous-trial certainty.

Analysis of the accuracy data as a function of certainty revealed a main effect of certainty $[F(1,23)=26.97, p<$ $.001]$, with participants being more accurate when they reported being sure than when they were unsure (see Table 1 and Fig. 2b). No main effect of previous-trial certainty $[F(1,23)=$ $0.13, p=.719$ ] or any interaction between previous- and current-trial certainty $[F(1,23)=0.25, p=.620]$ was found for the accuracy data. However, despite the absence of a PUIA effect on average, considerable variability in PUIA was present $(S D=19.00 \%)$, and this variability in PUIA correlated positively with PUS across participants $(r=.42, n=24, p=$ $.041)$. That is, the degree to which participants slowed their responding following correct-unsure responses was predictive of increases in accuracy (see Fig. 3).

When comparing errors and correct trials (collapsed across certainty), analysis of the RT data revealed a main effect of accuracy $[F(1,23)=33.00, p<.001]$, with participants responding more slowly when they made an error. However, no main effect of previous-trial accuracy $[F(1,23)=1.45, p=$ .240], or any interaction between previous- and current-trial accuracy $[F(1,23)=0.16, p=.696]$ was found for the RT data. Additionally, no evidence of a post-error increase in accuracy was identified $[t(1,23)=-0.90, p=.379]$.

An exploratory analysis of the RT data on a subset of the participants $(n=18)$, investigating the effects of both currentand previous-trial certainty and accuracy on the current-trial RT, revealed no two-, three-, or four-way interactions between certainty and accuracy (all $p s>.147$ ). However, as in the primary analysis of the behavioral data, a main effect of 

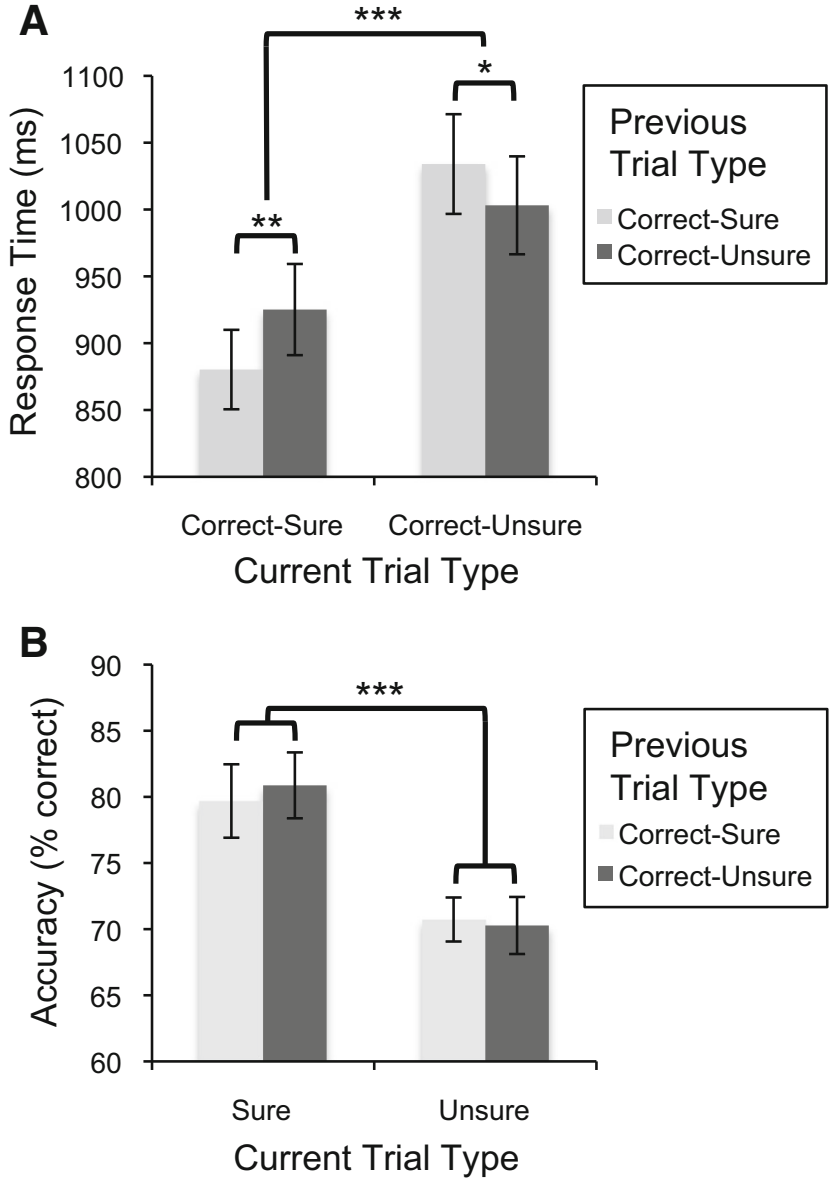

Fig. 2 Response time (RT) and accuracy data as a function of current and previous trial type. For all figures, significance values are denoted as $* p<$ $.05, * * p<.01, * * * p<.001$. a RT for correct trials only, as a function of current- and previous-trial certainty. A significant post-uncertainty slowing effect was found for sure trials that followed unsure trials. b Accuracy for sure and unsure responses, as a function of whether the previous (correct) trial was reported as sure or unsure

current-trial certainty was identified $[F(1,23)=26.85, p<$ $.001]$, as well as a current- by previous-trial certainty interaction $[F(1,23)=8.05, p=.011]$. The nature of this current- by previous-trial certainty interaction was similar to that interaction in the primary analysis, with the effect of previous-trial uncertainty eliciting slowing when the current trial produced a "sure" response $(M=968.51, S E=39.17$, vs. $M=927.62, S E$ $=34.61)$, and speeding when the current trial produced an "unsure" response $(M=1,036.07, S E=43.24$, vs. $M=1$, 069.26, $S E=48.33$ ), although these slowing and speeding effects were not individually significant in the reduced data set. Additionally, the inclusion of current- and previous-trial accuracy revealed both a main effect of current-trial accuracy $[F(1,23)=14.49, p=.001]$ and an interaction between current- and previous-trial accuracy $[F(1,23)=4.67, p=$ $.045]$. The nature of this current- by previous-trial interaction was similar to the contextual effects of certainty, with the effect of previous-trial errors eliciting slowing when the current trial produced a correct response $(M=986.64, S E=$

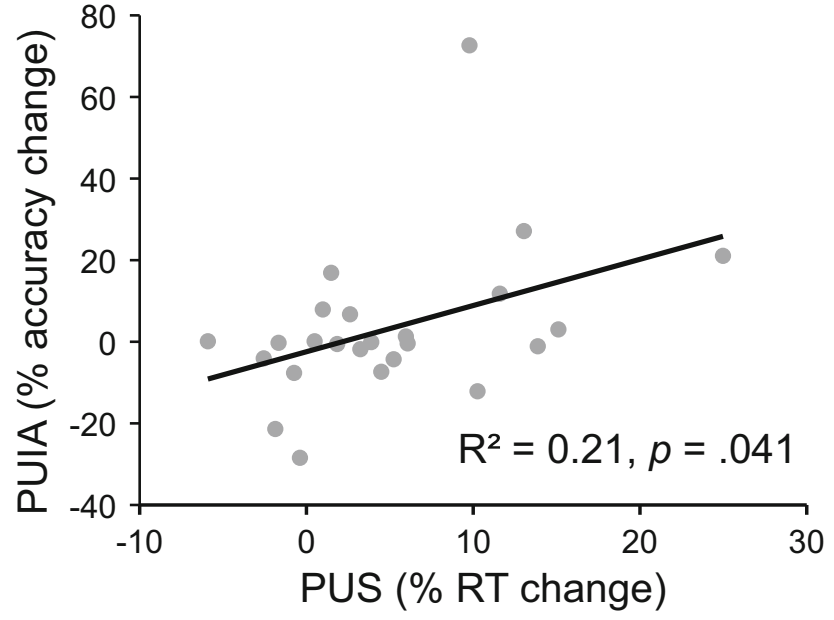

Fig. 3 Correlation between post-uncertainty slowing (PUS) and post-uncertainty increases in accuracy (PUIA). A significant positive correlation was identified between the percentage change in PUS and the percentage change in PUIA $(r=.42, p=.041)$. PUS was defined as the percentage change in RT for correct-sure trials following correctunsure trials, relative to each individual's baseline RT for correct-sure trials following correct-sure trials. PUIA was defined as the percentage change in accuracy for sure trials following correct-unsure trials, relative to each individual's baseline accuracy for sure trials following correctsure trials

39.76, vs. $M=970.33, S E=36.21)$, and speeding when the current trial produced an incorrect response $(M=1,016.64, S E$ $=41.37$, vs. $M=1,027.85, S E=42.12$ ), although the individual slowing and speeding effects were not significant.

The exploratory analysis investigating the effects of certainty and accuracy on current-trial accuracy (as an outcome measure) yielded a main effect of current-trial certainty $[F(1$, $23)=22.00, p<.001]$. However, no other significant effects were identified in this analysis (all $p s>.187$ ).

\section{Imaging results}

Primary analysis of imaging data The comparison of "sure" and "unsure" responses (for correct trials only) identified a dissociation between VS and MFC (see Fig. 4): Increased activation was identified within the VS for correct-sure trials, whereas increased MFC activation was identified for correctunsure trials. Specifically, a whole-brain analysis of the correct-sure $>$ correct-unsure contrast identified a single cluster (Cluster 1 in Table 2) with a peak on the border of right NAcc/subcallosal cortex, extending dorsally into ventral putamen and ventrally into orbitofrontal cortex (peak $Z=3.83$ at coordinates $8,10,-14 ; 614$ voxels). The correct-unsure $>$ correct-sure contrast also identified a single cluster (Cluster 2 in Table 2), with a peak in dorsal anterior cingulate cortex (dACC) extending dorsally into the presupplementary motor area and superior frontal gyrus (peak $Z=4.14$ at $-6,18,40$; 670 voxels). No other effects within the correct-sure versus correct-unsure contrasts survived correction for multiple 


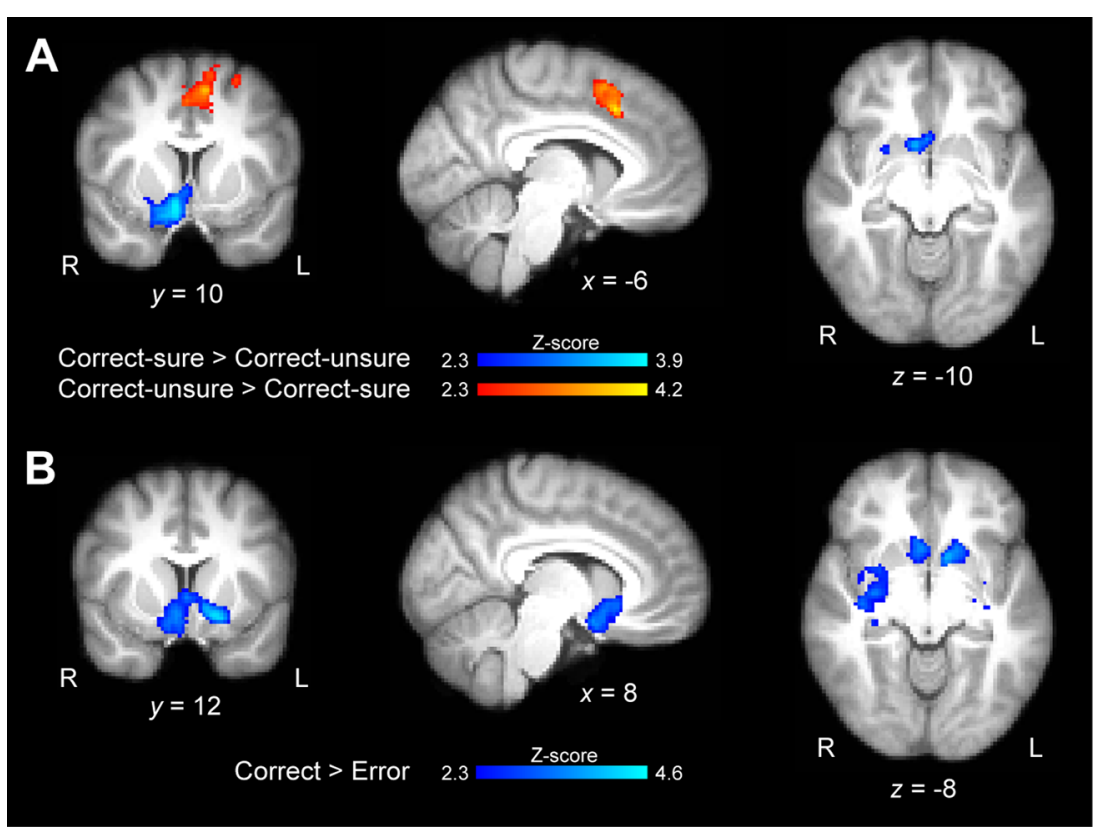

Fig. 4 Activation as a function of certainty and accuracy. a $Z$ maps reflecting the comparison of correct-sure and correct-unsure trials. From left to right: coronal $(y=10)$, sagittal $(x=-6)$, and axial $(z=-10)$ slices, cluster-corrected $(Z=2.3, p<.05)$. $\mathbf{b} Z$ maps reflecting the comparison of

comparisons; see Table 2 for a full list of the local maxima within the clusters identified.

The comparison of correct and incorrect responses (collapsed across certainty) revealed increased activation within the VS, as well as in right putamen and superior parietal lobule, for the correct responses (see Fig. 4). Specifically, a whole-brain analysis of the correct $>$ error contrast identified a large bilateral ventral striatal cluster (Cluster 3 in Table 2) extending into subcallosal cortex and orbitofrontal cortex (peak $Z=4.52$ at $-16,10,-12 ; 1,159$ voxels). Importantly, this cluster included bilateral NAcc, with the peak $Z$ value being located on the border of left NAcc and ventral putamen. Two additional clusters were identified within the correct $>$ error contrast. First, a large cluster (Cluster 4 in Table 2) with a peak in right posterolateral putamen, extending both laterally into the right temporal lobe and insula/opercular cortex, as well as ventrally into hippocampus/parahippocampal cortex (peak $Z$ $=3.41$ at $32,-10,-8 ; 1,236$ voxels). Second, a relatively small cluster (Cluster 5 in Table 2) with a peak in the left postcentral gyrus, extending into the superior parietal lobule and partially into the precentral gyrus (peak $Z=3.53$ at $-50,-20,54$; 460 voxels). No activations in the error $>$ correct contrast survived corrections for multiple comparisons. See Table 2 for a full list of the local maxima within the clusters identified.

\section{Relationship between uncertainty-dependent brain re-} gions and PUS The test for a correlation across participants between the uncertainty-dependent MFC ROI activity (for the correct-unsure $>$ correct-sure contrast) and PUS was found to be nonsignificant $(r=.128, n=24, p=.55)$. However, a robust correct and error trials (collapsed across certainty). From left to right: coronal $(y=12)$, sagittal $(x=8)$, and axial $(z=-8)$ slices, clustercorrected $(Z=2.3, p<.05)$. No activations survived correction for multiple comparisons in the error $>$ correct contrast

negative correlation between the uncertainty-dependent VS ROI activity (again for the correct-unsure> correct-sure contrast) and PUS was identified across participants $(r=-.581, n$ $=24, p=.002)$; greater reduction in VS activation for "unsure" responses (relative to "sure" responses) was associated with increased post-uncertainty slowing. See Fig. 5a and $\mathrm{b}$ for depictions of the MFC-PUS and VS-PUS relationships, respectively.

Within-subjects analysis of slowing effect A comparison of the slowing effects on a within-subjects basis revealed significantly reduced activation within the VS for unsure trials that were followed by greater slowing (see Fig. 6). Specifically, small-volume correction within the VS ROI of the unsureno-slowing $>$ unsure-slowing contrast identified a single cluster (Cluster 1 in Table 3) with a peak on the border of ventral putamen/orbitofrontal cortex and extending into the left NAcc (peak $Z=3.27$ at $-20,10,-12 ; 218$ voxels). Additionally, small-volume correction within the VS ROI of the sure-noslowing $>$ sure-slowing contrast identified a single cluster (Cluster 2 in Table 3) with a peak in subcallosal cortex, extending into orbitofrontal cortex and right NAcc (peak $Z$ $=3.77$ at $4,14,-16 ; 165$ voxels). No activations survived correction for multiple comparisons within the MFC ROI. See Table 3 for a full list of the local maxima within the clusters identified.

Exploratory analysis of the certainty by accuracy interaction The GLM modeling both the main effects of certainty and accuracy, as well as their interaction, identified main effects of 
Table 2 List of activations identified by contrasting certainty (for correct trials only) or by contrasting correct and error trials

\begin{tabular}{|c|c|c|c|c|c|c|c|}
\hline Anatomical Area & Cluster & Side & $x$ & $y$ & $z$ & $Z \max$ & Voxels \\
\hline \multicolumn{8}{|l|}{ Correct-sure $>$ Correct-unsure } \\
\hline Subcallosal cortex, NAcc & 1 & $\mathbf{R}$ & 8 & 10 & -14 & 3.83 & \multirow[t]{6}{*}{614} \\
\hline $\mathrm{OFC}$ & 1 & $\mathrm{R}$ & 20 & 12 & -20 & 3.51 & \\
\hline Subcallosal cortex & 1 & $\mathrm{R}$ & 2 & 14 & -10 & 3.16 & \\
\hline $\mathrm{OFC}$ & 1 & $\mathrm{R}$ & 24 & 22 & -26 & 3.02 & \\
\hline Amygdala & 1 & $\mathrm{R}$ & 24 & -2 & -14 & 3.01 & \\
\hline OFC & 1 & $\mathrm{R}$ & 22 & 28 & -28 & 2.88 & \\
\hline \multicolumn{8}{|l|}{ Correct-unsure $>$ Correct-sure } \\
\hline dACC (BA 32') & 2 & $\mathbf{L}$ & -6 & 18 & 40 & 4.14 & \multirow[t]{6}{*}{670} \\
\hline pre-SMA (medial BA 6) & 2 & $\mathrm{~L}$ & -8 & 10 & 52 & 3.71 & \\
\hline Superior frontal gyrus & 2 & $\mathrm{~L}$ & -24 & -6 & 50 & 3.56 & \\
\hline dACC (BA 32) & 2 & $\mathrm{~L}$ & -8 & 18 & 50 & 3.34 & \\
\hline dACC (BA 32') & 2 & $\mathrm{~L}$ & -2 & 8 & 50 & 3.27 & \\
\hline dACC (BA 32) & 2 & $\mathrm{R}$ & 8 & 16 & 38 & 3.11 & \\
\hline \multicolumn{8}{|l|}{ Correct $>$ Error } \\
\hline Putamen, NAcc & 3 & $\mathbf{L}$ & -16 & 10 & -12 & 4.52 & \multirow[t]{6}{*}{1,159} \\
\hline OFC & 3 & $\mathrm{R}$ & 16 & 8 & -16 & 3.99 & \\
\hline Subcallosal cortex & 3 & $\mathrm{R}$ & 4 & 10 & -18 & 3.91 & \\
\hline Subcallosal cortex & 3 & $\mathrm{R}$ & 2 & 16 & -2 & 3.75 & \\
\hline Subcallosal cortex, NAcc & 3 & $\mathrm{R}$ & 6 & 12 & -12 & 3.54 & \\
\hline Amygdala & 3 & $\mathrm{~L}$ & -18 & -2 & -16 & 3.01 & \\
\hline Putamen & 4 & $\mathbf{R}$ & 32 & -10 & -8 & 3.41 & \multirow[t]{6}{*}{1,236} \\
\hline Hippocampus & 4 & $\mathrm{R}$ & 32 & -28 & -12 & 3.4 & \\
\hline Cerebral white matter, Planum polare & 4 & $\mathrm{R}$ & 38 & -16 & -10 & 38 & \\
\hline Heschl's gyrus, Insular cortex & 4 & $\mathrm{R}$ & 44 & -12 & 4 & 3.24 & \\
\hline Hippocampus & 4 & $\mathrm{R}$ & 28 & -18 & -16 & 3.2 & \\
\hline Planum temporal & 4 & $\mathrm{R}$ & 60 & -20 & 8 & 3.14 & \\
\hline Postcentral gyrus & 5 & $\mathbf{L}$ & $-\mathbf{5 0}$ & -20 & 54 & 3.53 & \multirow[t]{6}{*}{460} \\
\hline Postcentral gyrus, Superior parietal lobule & 5 & $\mathrm{~L}$ & -40 & -34 & 52 & 3.48 & \\
\hline Superior parietal lobule, Postcentral gyrus & 5 & $\mathrm{~L}$ & -42 & -42 & 60 & 3.34 & \\
\hline Postcentral gyrus & 5 & $\mathrm{~L}$ & -30 & -28 & 68 & 3.28 & \\
\hline Precentral gyrus & 5 & $\mathrm{~L}$ & -30 & -24 & 68 & 3.27 & \\
\hline Postcentral gyrus & 5 & $\mathrm{~L}$ & -48 & -32 & 56 & 3.21 & \\
\hline \multicolumn{8}{|l|}{ Error $>$ Correct } \\
\hline- & - & - & - & - & - & - & - \\
\hline
\end{tabular}

$B A$ Brodmann area, $d A C C$ dorsal anterior cingulate cortex, $N A c c$ nucleus accumbens cortex, $O F C$ orbitofrontal cortex, pre-SMA pre-supplementary motor area

certainty and accuracy similar to those identified within the primary analysis of the imaging data (see Fig. 7). Specifically, small-volume correction within the VS ROI of the sure $>$ unsure contrast identified a cluster (Cluster 1 in Table 4) with a peak on the border of subcallosal cortex and right NAcc, extending into orbitofrontal cortex (peak $Z=3.54$ at $8,10,-14$; 232 voxels), as well as a second cluster (Cluster 2 in Table 4) with a peak on the border of orbitofrontal cortex and left NAcc, extending into subcallosal cortex and ventral putamen (peak $Z=3.74$ at $-10,8,-16 ; 176$ voxels). Small-volume correction within the MFC ROI of the unsure $>$ sure contrast identified a single cluster (Cluster 3 in Table 4) with a peak on the border of dACC, superior frontal gyrus, and presupplementary motor area (peak $Z=3.85$ at $-4,16,46$; 159 voxels). Small-volume correction within the VS ROI of the correct $>$ error contrast identified a single cluster spanning bilateral VS (Cluster 4 in Table 4) with a peak on the border of right ventral putamen and NAcc, extending into left NAcc, as well as subcallosal cortex and orbitofrontal cortex (peak $Z=3.63$ at $-16,12,-12$; 482 voxels). However, no significant activation was identified for the error $>$ correct contrast, nor any interaction between accuracy and certainty identified. See Table 4 for a full list of the local maxima within the clusters identified. 


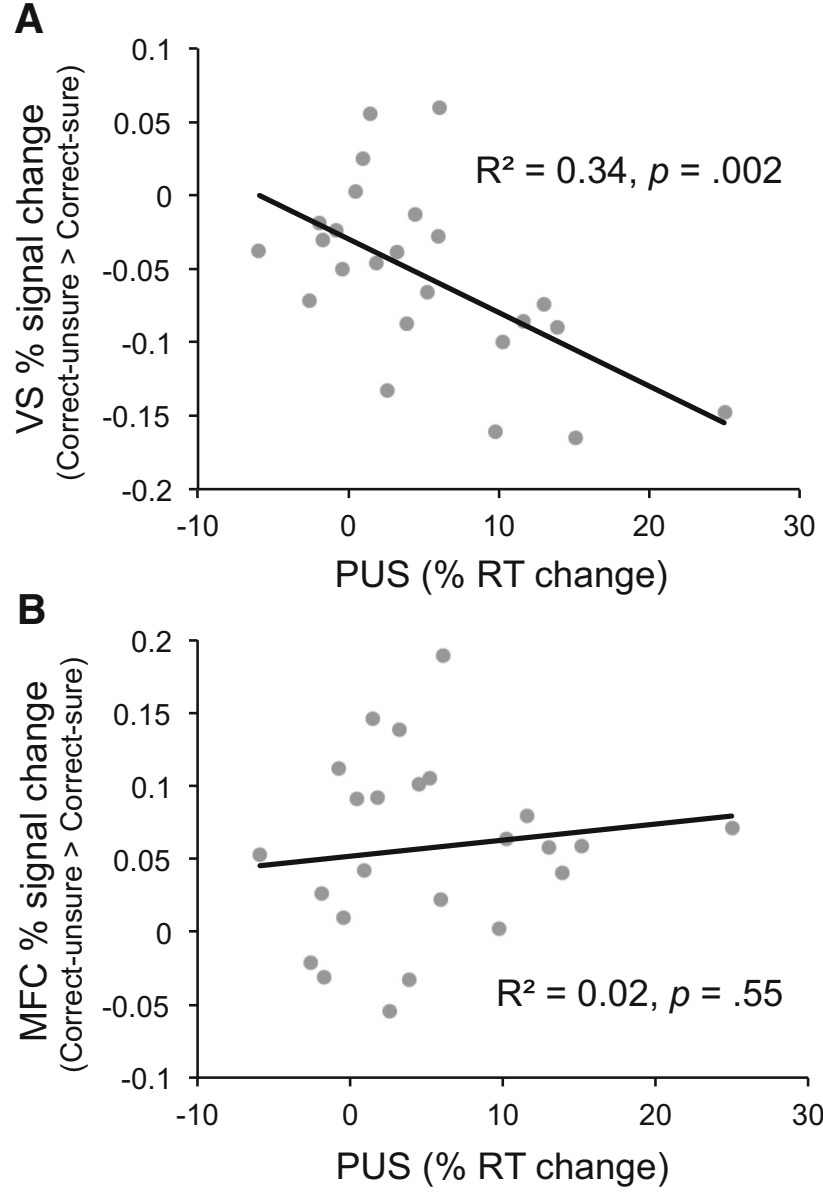

Fig. 5 Correlations between post-uncertainty slowing (PUS) and uncertainty-dependent medial frontal cortex (MFC) and ventral striatum (VS) activity. For all figures, PUS was defined as each participant's average percentage change in RT for correct-sure trials following correct-unsure trials, relative to the participant's average baseline RT for correct-sure trials following correct-sure trials. a PUS negatively correlated with percent signal change in the VS ROI $(r=-.581, p=$ $.002)$. The VS percent signal change reflects the mean percent signal change for each participant, extracted from the correct-unsure $>$ correctsure contrast. b PUS did not correlate with percent signal change in the MFC ROI $(r=.128, p=.55)$. The MFC percent signal change reflects the mean percent signal change for each participant, extracted from the correct-unsure > correct-sure contrast

\section{Discussion}

In the present study, we employed a difficult perceptual decision-making task in order to investigate whether uncertainty, in the absence of feedback, can lead to task-related behavioral adaptations. In line with our predictions, uncertain responses led to response slowing on subsequent trials, a phenomenon that was correlated across participants with increases in accuracy. This finding suggests that instances of perceptual uncertainty can trigger a more cautious response strategy in order to achieve task goals. Critically, the extent of PUS was correlated across participants with the magnitude of uncertainty-dependent modulations within the VS. Additionally, the relationship between VS activity and PUS was present at the within-subjects level, with reduced VS activation on unsure trials being predictive of slowing on the subsequent trial. This finding supports the notion that confidence is encoded within VS, providing an "intrinsic reinforcement signal" that guides ongoing behavior (Hebart et al., 2014); when this signal is reduced on uncertain trials, a change in response strategy is engaged as a result. In agreement with previous work, we also found that perceptual uncertainty was associated with both increased MFC activity and reduced VS activity (Fleck et al., 2006; Fleming et al., 2012; Hebart et al., 2014), although MFC activation was not correlated with PUS. VS activity was also increased for correct responses (as compared to errors), in line with recent work suggesting that correct responses may be intrinsically rewarding (Satterthwaite et al., 2012; Schwarze et al., 2013).

We measured post-trial RT and accuracy as indices of behavioral adaptations following uncertain responses. This approach is similar to that used by previous studies investigating PES and PEIA (for a review, see Danielmeier \& Ullsperger, 2011). We identified a novel effect of PUS, which was positively correlated with PUIA across participants. This suggests that slowing on trials following uncertain responses is adaptive (and not simply an index of distraction), since it leads to increased accuracy. We interpret this PUS effect within the

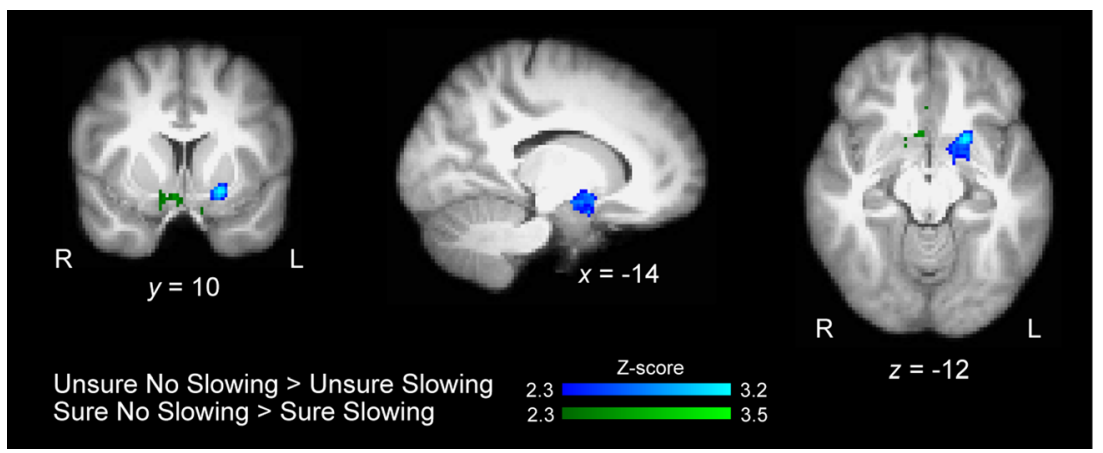

Fig. 6 Activation for the within-subjects analysis of slowing effects: $Z$ maps reflecting the slowing effects for correct-sure and correct-unsure trials. From left to right: coronal $(y=10)$, sagittal $(x=-14)$, and axial $(z$
$=-12)$ slices, small-volume cluster-corrected $(Z=2.3, p<.05)$ within VS and MFC 20-mm spheres 
Table 3 List of activations identified by separately contrasting sure and unsure trials, with and without slowing

\begin{tabular}{|c|c|c|c|c|c|c|c|}
\hline Anatomical Area & Cluster & Hem & $x$ & $y$ & $z$ & $Z \max$ & Voxels \\
\hline \multicolumn{8}{|c|}{ Correct-unsure no slowing > Correct-unsure slowing } \\
\hline Putamen, OFC & 1 & $\mathbf{L}$ & -20 & 10 & -12 & 3.27 & 218 \\
\hline OFC & 1 & $\mathrm{~L}$ & -14 & 4 & -20 & 2.85 & \\
\hline White matter & 1 & $\mathrm{~L}$ & -14 & 0 & -14 & 2.80 & \\
\hline Pallidum & 1 & $\mathrm{~L}$ & -14 & 4 & -10 & 2.78 & \\
\hline Amygdala & 1 & $\mathrm{~L}$ & -14 & -4 & -14 & 2.75 & \\
\hline Putamen & 1 & $\mathrm{~L}$ & -18 & 2 & -10 & 2.74 & \\
\hline \multicolumn{8}{|c|}{ Correct-sure no slowing $>$ Correct-sure slowing } \\
\hline Subcallosal cortex & 2 & $\mathbf{R}$ & 4 & 14 & -16 & 3.77 & 165 \\
\hline Subcallosal cortex & 2 & $\mathrm{~L}$ & -2 & 18 & -20 & 3.36 & \\
\hline Subcallosal cortex & 2 & $\mathrm{~L}$ & -8 & 16 & -18 & 2.77 & \\
\hline OFC & 2 & $\mathrm{R}$ & 12 & 8 & -18 & 2.76 & \\
\hline Subcallosal cortex & 2 & $\mathrm{R}$ & 2 & 28 & -14 & 2.50 & \\
\hline OFC & 2 & $\mathrm{~L}$ & -10 & 10 & -24 & 2.37 & \\
\hline
\end{tabular}

OFC orbitofrontal cortex

context of previous work supporting a control theory of PES; these studies suggest that PES is the result of an increased motor threshold that allows for increased cautiousness (Botvinick et al., 2001; Ratcliff \& McKoon, 2008). Although it has also been suggested that PES may simply reflect distraction (Notebaert et al., 2009), it is important to note that the functional role of PES may differ depending on the ISI used in an experiment (Danielmeier \& Ullsperger, 2011; Dudschig \& Jentzsch, 2009; Jentzsch \& Dudschig, 2009). When trials are separated by longer ISIs, as was the case in the present experiment, any distraction or orienting associated with errors (or, in the present case, uncertainty) should have time to be translated into a change in response strategy. We also note that the suggestion that PES may reflect distraction

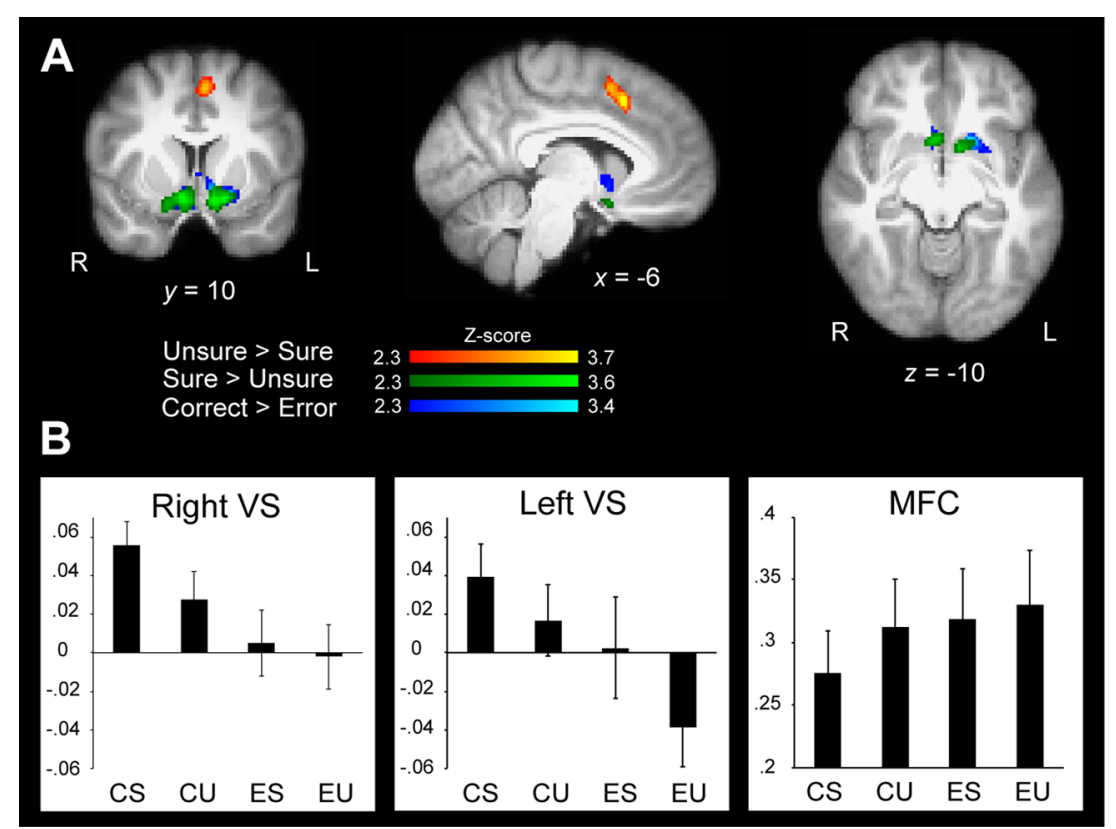

Fig. 7 Activation for the 2 by 2 analysis of certainty and accuracy. a $Z$ maps reflecting the main effects of certainty and accuracy. From left to right: coronal $(y=10)$, sagittal $(x=-6)$, and axial $(z=-10)$ slices. No activations survived correction for multiple comparisons in the error $>$ correct contrast, and no interaction between certainty and accuracy was identified. The results reflect small-volume cluster correction $(Z=2.3, p$ $<.05)$ within VS and MFC. b Plots of percent signal change within the right VS (6-mm sphere centered on right NAcc), left VS (6-mm sphere centered on left NAcc), and MFC (6-mm sphere centered on the region of MFC sensitive to performance monitoring, as defined by the metaanalysis of Klein et al., 2007) for correct-sure (CS), correct-unsure $(\mathrm{CU})$, error-sure (ES), and error-unsure (EU) trials. Error bars reflect the standard errors of the means 
Table 4 List of activations identified by the ANOVA model testing for main effects of certainty and accuracy, as well as their interaction

\begin{tabular}{|c|c|c|c|c|c|c|c|}
\hline Anatomical Area & Cluster & Side & $x$ & $y$ & $z$ & $Z \max$ & Voxels \\
\hline \multicolumn{8}{|l|}{ Sure $>$ Unsure } \\
\hline Subcallosal cortex, NAcc & 1 & $\mathbf{R}$ & 8 & 10 & -14 & 3.54 & \multirow[t]{3}{*}{232} \\
\hline OFC & 1 & $\mathrm{R}$ & 16 & 10 & -18 & 2.88 & \\
\hline OFC & 1 & $\mathrm{R}$ & 22 & 14 & -22 & 2.51 & \\
\hline Subcallosal cortex, OFC, NAcc & 2 & $\mathbf{L}$ & -10 & 8 & -16 & 3.74 & \multirow[t]{2}{*}{176} \\
\hline OFC, Putamen, NAcc & 2 & $\mathrm{~L}$ & -16 & 10 & -14 & 3.56 & \\
\hline \multicolumn{8}{|l|}{ Unsure $>$ Sure } \\
\hline dACC (BA 32'), Superior frontal gyrus, pre-SMA & 3 & $\mathbf{L}$ & -4 & 16 & 46 & 3.85 & 159 \\
\hline \multicolumn{8}{|l|}{ Correct $>$ Error } \\
\hline Putamen, OFC, Subcallosal cortex, NAcc & 4 & $\mathbf{L}$ & -16 & 12 & -12 & 3.63 & \multirow[t]{6}{*}{482} \\
\hline Subcallosal cortex & 4 & $\mathrm{R}$ & 4 & 4 & -18 & 3.42 & \\
\hline Hippocampus, Amygdala & 4 & $\mathrm{~L}$ & -14 & -14 & -16 & 3.35 & \\
\hline NAcc & 4 & $\mathrm{R}$ & 4 & 4 & -2 & 3.31 & \\
\hline White matter & 4 & $\mathrm{~L}$ & -10 & -10 & -8 & 3.26 & \\
\hline Orbitofrontal cortex & 4 & $\mathrm{R}$ & 16 & 16 & -16 & 2.98 & \\
\hline \multicolumn{8}{|l|}{ Error $>$ Correct } \\
\hline- & - & - & - & - & - & - & - \\
\hline \multicolumn{8}{|l|}{ Certainty*Accuracy } \\
\hline- & - & - & - & - & - & - & - \\
\hline
\end{tabular}

$B A$ Brodmann area, $d A C C$ dorsal anterior cingulate cortex, $N A c c$ nucleus accumbens cortex, $O F C$ orbitofrontal cortex, pre-SMA pre-supplementary motor area

following errors is based, in part, on the observation that errors are typically infrequent (and therefore salient) events that capture attention (Notebaert et al., 2009). However, the degree of PUS did not correlate with the frequency of uncertain responses across participants (see the supplementary material), suggesting that PUS was not a result of the frequency of uncertain events. Thus, we interpret PUS (and the associated increases in accuracy) as an index of control.

It should be noted that although we focused primarily on the slowing effect that follows unsure responses, a speeding effect was also observed when unsure responses were followed by a subsequent unsure response. This pattern of results is very similar to the well-known congruency sequence effect (also termed the Gratton effect) typically observed in tasks involving externally induced response (Gratton, Coles, \& Donchin, 1992; Kerns et al., 2004; Stürmer, Leuthold, Soetens, Schröter, \& Sommer, 2002) or stimulus (Verbruggen, Notebaert, Liefooghe, \& Vandierendonck, 2006) conflict. In such tasks involving externally induced conflict, the RTs for incongruent trials (those involving conflict) are generally faster when the trial is preceded by another incongruent trial, as compared to when an incongruent trial is preceded by a congruent trial. Similarly, in the present investigation we found that unsure responses were generally faster when they were preceded by another unsure response, relative to when an unsure response was preceded by a sure response. Traditionally, the observation of a congruency sequence effect has been interpreted as an index of cognitive control, on the basis of the assumption that detecting conflict leads to an up-regulation of cognitive control on the next trial (Botvinick et al., 2001). Although it has also been suggested that congruency sequence effects can reflect low-level memory effects related to a stimulus-response binding process (see Hommel, Proctor, \& $\mathrm{Vu}, 2004$, for a detailed explanation), several reports have shown evidence for both the cognitive-control and low-level accounts of the congruency sequence effect occurring in parallel within the same task (for reviews, see Duthoo, Abrahamse, Braem, Boehler, \& Notebaert, 2014; Egner, 2007). In the present investigation, the functional interpretation of the observed congruency sequence effect remains unclear, since neither the speeding effect nor the congruency sequence effect overall was significantly related to either accuracy or VS changes (see the supplementary material). However, strong support for the PUS effect reflecting control stems from a positive correlation with accuracy, as well as the associated negative correlation with VS activity on the previous trial. Thus, although our data provide evidence for a congruency sequence effect as a function of self-reported uncertainty, it appears that a separable PUS effect reflects a more direct index of cognitive control following unsure responses.

The observed association between PUS and VS activation suggests a possible neural mechanism for task-positive behavioral adaptations following instances of uncertainty. An association between confidence-related VS activity and behavior was previously suggested by Hebart et al. (2014), but not directly observed. These authors demonstrated that the VS encodes a choice-independent confidence signal following perceptual decisions. Although the focus of their report was 
on establishing the existence of this signal in relation to decision processes, they suggested that the function of a confidence signal might be to adapt ongoing behavior. This suggestion was also supported by previous work showing that VS activity for correct responses (in the absence of feedback) scales with the task difficulty and predicts overall task performance in a working memory paradigm (Satterthwaite et al., 2012). Here, we confirmed this speculation by showing that uncertainty-dependent modulation of activity within the VS predicts the degree to which participants adapt their behavior throughout a task. More generally, the relationship between VS and task-positive changes in behavior is in line with a large literature associating the VS with reward and motivation (for reviews, see Delgado, 2007; Shohamy, 2011). For example, it has been demonstrated that VS activity correlates with the effort put forward to gain a reward (Schmidt et al., 2012). The present findings extend previous work on the association between VS and behavioral adaptations to situations in which no external rewards or feedback are present. Although the exact mechanism by which VS activity is translated into behavioral adaptations remains unclear, the present data support a strong relationship between the VS and PUS.

In contrast to the observed PUS effect, and the associated PUIA, we did not observe evidence for classical PES or PEIA in the full behavioral data set. Although an interaction between current- and previous-trial accuracy on RT was observed in the exploratory analysis of a subset of participants $(n=18)$, the slowing effect remained nonsignificant. However, the absence of a PES effect is not surprising, because for the present study we employed a highly difficult perceptual task in which selfdetection of errors was limited. In the present study, both errors and correct responses were associated with a high degree of uncertainty, creating a context in which it was possible for participants only to detect when they were "unsure" of their accuracy, but not explicitly "aware" of their accuracy. In line with this notion, MFC activity, which is typically observed on error trials, was absent in the error > correct comparison of the primary analysis of our imaging data. It should also be noted that in the exploratory analysis of the RT data, the effect of previous-trial certainty did not interact with the previous-trial accuracy. Similarly, in the exploratory analysis of the imaging data, uncertainty and accuracy did not interact, and MFC activity was observed for the main effect of certainty but not of accuracy. These findings reinforce the interpretation that uncertainty alone, and not objective accuracy, drove both MFC activity and post-trial slowing in the present task.

In addition to an association between PUS and VS, we also hypothesized a potential relationship between PUS and MFC activity. Such a link between the MFC and post-trial adaptations is in line with a general notion that the primary function of the MFC is to decide when and how to adapt behavior (Miller \& Cohen, 2001; Ridderinkhof, Ullsperger, Crone, \& Nieuwenhuis, 2004; Shenhav et al., 2013). Although we did not observe an MFC-PUS relationship in the present experiment, a strong relationship between PUS and another key node in the performance-monitoring network, the VS, was observed. The VS-PUS relationship is in line with at least two dominant theories of the MFC - the "expected value of control" theory (Shenhav et al., 2013) and reinforcement-learning theory (Holroyd \& Coles, 2002) - which describe the importance of VS input to the MFC for deciding when and how to adapt behavior. Additionally, the VS receives extensive input from several structures, including the MFC, and has access to both cognitive and motor regions through projections to the cholinergic system of the nucleus basalis and the globus pallidus (Haber \& Knutson, 2010). Thus, we suggest that uncertaintyrelated changes in behavior may be more closely tied to the VS than to the MFC. However, whether this phenomenon is unique to perceptual uncertainty, or can be generalized to other forms of uncertainty, remains to be determined.

\section{Conclusions and future directions}

The present study provides evidence that perceptual uncertainty triggers behavioral adaptations on subsequent trials. Specifically, participants were more likely to slow down following unsure than following sure responses, and this slowing correlated positively with post-uncertainty increases in accuracy. Critically, post-uncertainty slowing was correlated with the degree to which VS activity was modulated by uncertainty on the previous trial. This suggests that a confidence signal encoded within the VS is used to adaptively influence behavior. Future work should seek to investigate the cascade of neural activity that translates this confidence signal encoded within the VS into trial-by-trial adaptations of behavior. Additionally, whereas the present study focused on the relationship between perceptual uncertainty and behavioral adaptations, future work should also investigate the relationship between other types of uncertainty (such as outcome uncertainty) and ongoing behavioral adaptations.

Author note This research was supported by the Air Force Office of Scientific Research, Grant Number FA9550-10-1-0385, the Center of Excellence in Neuroergonomics, Technology, and Cognition.

\section{References}

Botvinick, M. M. (2007). Conflict monitoring and decision making: Reconciling two perspectives on anterior cingulate function. Cognitive, Affective, \& Behavioral Neuroscience, 7, 356-366. doi: 10.3758/CABN.7.4.356

Botvinick, M. M., Braver, T. S., Barch, D. M., Carter, C. S., \& Cohen, J. D. (2001). Conflict monitoring and cognitive control. Psychological Review, 108, 624-652. doi:10.1037/0033-295X.108.3.624

Brainard, D. H. (1997). The psychophysics toolbox. Spatial Vision, 10, 433-436. doi:10.1163/156856897X00357 
Chevrier, A., \& Schachar, R. J. (2010). Error detection in the stop signal task. NeuroImage, 53, 664-673. doi:10.1016/j.neuroimage.2010.06.056

Clos, M., Schwarze, U., Gluth, S., Bunzeck, N., \& Sommer, T. (2015). Goal- and retrieval-dependent activity in the striatum during memory recognition. Neuropsychologia, 72, 1-11. doi:10.1016/j. neuropsychologia.2015.04.011

Danielmeier, C., Eichele, T., Forstmann, B. U., Tittgemeyer, M., \& Ullsperger, M. (2011). Posterior medial frontal cortex activity predicts post-error adaptations in task-related visual and motor areas. Journal of Neuroscience, 31, 1780-1789. doi:10.1523/JNEUROSCI.4299-10.2011

Danielmeier, C., \& Ullsperger, M. (2011). Post-error adjustments. Frontiers in Psychology, 2, 233. doi:10.3389/fpsyg.2011.00233

Delgado, M. R. (2007). Reward-related responses in the human striatum. Annals of the New York Academy of Sciences, 1104, 70-88. doi:10. 1196/annals.1390.002

Dreisbach, G., \& Fischer, R. (2012). Conflicts as aversive signals. Brain and Cognition, 78, 94-98. doi:10.1016/j.bandc.2011.12.003

Dudschig, C., \& Jentzsch, I. (2009). Speeding before and slowing after errors: Is it all just strategy? Brain Research, 1296, 56-62. doi:10. 1016/j.brainres.2009.08.009

Duthoo, W., Abrahamse, E. L., Braem, S., Boehler, C. N., \& Notebaert, W. (2014). The heterogeneous world of congruency sequence effects: An update. Frontiers in Psychology, 5, 1001. doi:10.3389/ fpsyg.2014.01001

Dutilh, G., Vandekerckhove, J., Forstmann, B. U., Keuleers, E., Brysbaert, M., \& Wagenmakers, E.-J. (2011). Testing theories of post-error slowing. Attention, Perception, \& Psychophysics, 74, 454-465. doi:10.3758/s13414-011-0243-2

Egner, T. (2007). Congruency sequence effects and cognitive control. Cognitive, Affective, \& Behavioral Neuroscience, 7, 380-390. doi: 10.3758/CABN.7.4.380

Fleck, M. S., Daselaar, S. M., Dobbins, I. G., \& Cabeza, R. (2006). Role of prefrontal and anterior cingulate regions in decision-making processes shared by memory and nonmemory tasks. Cerebral Cortex, 16, 1623-1630. doi:10.1093/cercor/bhj097

Fleming, S. M., Huijgen, J., \& Dolan, R. J. (2012). Prefrontal contributions to metacognition in perceptual decision making. Journal of Neuroscience, 32, 6117-6125. doi:10.1523/JNEUROSCI.6489-11.2012

Garavan, H., Ross, T. J., Murphy, K., Roche, R. A. P., \& Stein, E. A. (2002). Dissociable executive functions in the dynamic control of behavior: Inhibition, error detection, and correction. NeuroImage, 17, 1820-1829. doi:10.1006/nimg.2002.1326

Gratton, G., Coles, M. G. H., \& Donchin, E. (1992). Optimizing the use of information: Strategic control of activation of responses. Journal of Experimental Psychology. General, 121, 480-506. doi:10.1037/ 0096-3445.121.4.480

Groenewegen, H. J., Wright, C. I., \& Beijer, A. V. J. (1996). The nucleus accumbens: Gateway for limbic structures to reach the motor system? In G. Holstege, R. Bandler, \& C. B. Saper (Eds.), Progress in brain research: The emotional motor system (Vol. 107, pp. 485511). Amsterdam: Elsevier. doi:10.1016/S0079-6123(08)61883-X

Haber, S. N., \& Knutson, B. (2010). The reward circuit: Linking primate anatomy and human imaging. Neuropsychopharmacology, 35, 4 26. doi:10.1038/npp.2009.129

Hebart, M. N., Schriever, Y., Donner, T. H., \& Haynes, J.-D. (2014). The relationship between perceptual decision variables and confidence in the human brain. Cerebral Cortex. doi:10.1093/cercor/bhu181

Holroyd, C. B., \& Coles, M. G. H. (2002). The neural basis of human error processing: Reinforcement learning, dopamine, and the errorrelated negativity. Psychological Review, 109, 679-709. doi:10. 1037/0033-295X.109.4.679

Hommel, B., Proctor, R. W., \& Vu, K.-P. L. (2004). A feature-integration account of sequential effects in the Simon task. Psychological Research, 68, 1-17. doi:10.1007/s00426-003-0132-y

Jentzsch, I., \& Dudschig, C. (2009). Why do we slow down after an error? Mechanisms underlying the effects of posterror slowing.
Quarterly Journal of Experimental Psychology, 62, 209-218. doi: 10.1080/17470210802240655

Kerns, J. G., Cohen, J. D., MacDonald, A. W., Cho, R. Y., Stenger, V. A., \& Carter, C. S. (2004). Anterior cingulate conflict monitoring and adjustments in control. Science, 303, 1023-1026. doi:10.1126/ science. 1089910

King, J. A., Korb, F. M., von Cramon, D. Y., \& Ullsperger, M. (2010). Post-error behavioral adjustments are facilitated by activation and suppression of task-relevant and task-irrelevant information processing. Journal of Neuroscience, 30, 12759-12769. doi:10.1523/ JNEUROSCI.3274-10.2010

Kleiner, M., Brainard, D., \& Pelli, D. (2007). What's new in Psychtoolbox-3? Perception, 36(ECVP Abstract Supplement).

Klein, T. A., Endrass, T., Kathmann, N., Neumann, J., von Cramon, D. Y., \& Ullsperger, M. (2007). Neural correlates of error awareness. NeuroImage, 34, 1774-1781. doi:10.1016/j.neuroimage.2006.11.014

Miller, E. K., \& Cohen, J. D. (2001). An integrative theory of prefrontal cortex function. Annual Review of Neuroscience, 24, 167-202. doi: 10.1146/annurev.neuro.24.1.167

Murayama, K., Matsumoto, M., Izuma, K., \& Matsumoto, K. (2010). Neural basis of the undermining effect of monetary reward on intrinsic motivation. Proceedings of the National Academy of Sciences, 107, 20911-20916. doi:10.1073/pnas.1013305107

Notebaert, W., Houtman, F., Opstal, F. V., Gevers, W., Fias, W., \& Verguts, T. (2009). Post-error slowing: An orienting account. Cognition, 111, 275-279. doi:10.1016/j.cognition.2009.02.002

Pelli, D. G. (1997). The VideoToolbox software for visual psychophysics: Transforming numbers into movies. Spatial Vision, 10, 437-442. doi:10.1163/156856897X00366

Rabbitt, P. M., \& Phillips, S. (1967). Error-detection and correction latencies as a function of S-R compatibility. Quarterly Journal of Experimental Psychology, 19, 37-42. doi:10.1080/14640746708400065

Ratcliff, R., \& McKoon, G. (2008). The diffusion decision model: Theory and data for two-choice decision tasks. Neural Computation, 20, 873-922. doi:10.1162/neco.2008.12-06-420

Ridderinkhof, K. R., Ullsperger, M., Crone, E. A., \& Nieuwenhuis, S. (2004). The role of the medial frontal cortex in cognitive control. Science, 306, 443-447. doi:10.1126/science.1100301

Satterthwaite, T. D., Ruparel, K., Loughead, J., Elliott, M. A., Gerraty, R. T., Calkins, M. E., \& Wolf, D. H. (2012). Being right is its own reward: Load and performance related ventral striatum activation to correct responses during a working memory task in youth. NeuroImage, 61, 723-729. doi:10.1016/j.neuroimage.2012.03.060

Schmidt, L., Lebreton, M., Cléry-Melin, M.-L., Daunizeau, J., \& Pessiglione, M. (2012). Neural mechanisms underlying motivation of mental versus physical effort. PLoS Biology, 10, e1001266. doi: 10.1371/journal.pbio. 1001266

Schwarze, U., Bingel, U., Badre, D., \& Sommer, T. (2013). Ventral striatal activity correlates with memory confidence for old- and new-responses in a difficult recognition test. PLOS ONE, 8, e54324. doi:10.1371/journal.pone.0054324

Shenhav, A., Botvinick, M. M., \& Cohen, J. D. (2013). The expected value of control: An integrative theory of anterior cingulate cortex function. Neuron, 79, 217-240. doi:10.1016/j.neuron.2013.07.007

Shohamy, D. (2011). Learning and motivation in the human striatum. Current Opinion in Neurobiology, 21, 408-414. doi:10.1016/j. conb.2011.05.009

Stürmer, B., Leuthold, H., Soetens, E., Schröter, H., \& Sommer, W. (2002). Control over location-based response activation in the Simon task: Behavioral and electrophysiological evidence. Journal of Experimental Psychology. Human Perception and Performance, 28, 1345-1363. doi:10.1037/0096-1523.28.6.1345

Verbruggen, F., Notebaert, W., Liefooghe, B., \& Vandierendonck, A. (2006). Stimulus- and response-conflict-induced cognitive control in the flanker task. Psychonomic Bulletin \& Review, 13, 328-333. doi:10.3758/BF03193852 